\title{
Nonlinear Predictive Control With End Point Constraints
}

\author{
R. Hedjar* \\ Computer Engineering Department, College of Computer and Information Sciences - King Saud University, \\ P.O.Box 51178- Riyadh 11543, Saudi Arabia
}

Received 8 May 2005 ; accepted 16 November 2005

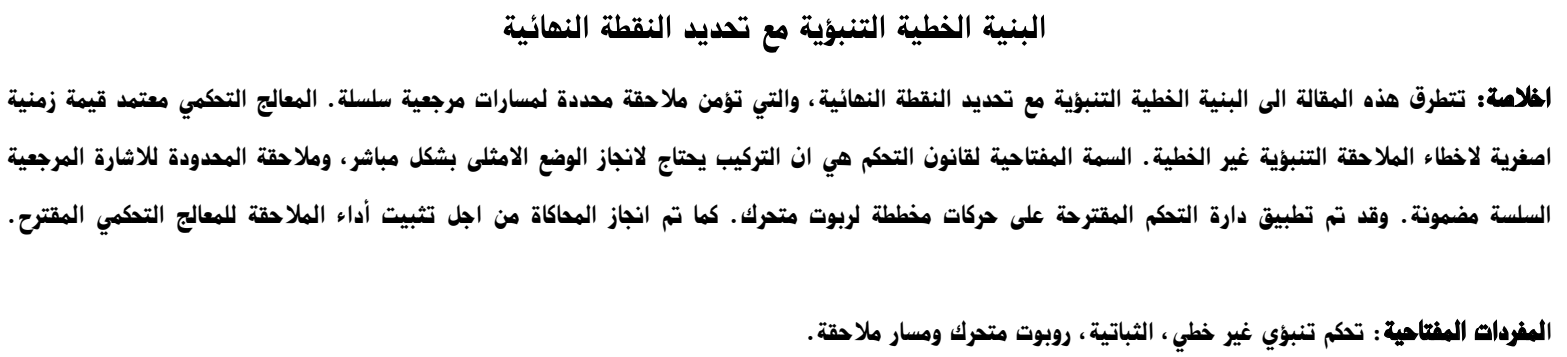

\begin{abstract}
The optimal nonlinear predictive control structure with end point constraints is presented, which provides asymptotic tracking of smooth reference trajectories. The controller is based on a finite horizon continuous time minimization of nonlinear predicted tracking errors. A key feature of the control law is that its implementation does not need to perform an online optimization, and asymptotic tracking of smooth reference signal is guaranteed. The proposed control scheme is applied to planning motions problem of a mobile robot. Simulations results are performed to validate the tracking performance of the proposed controller.
\end{abstract}

Keywords: Nonlinear predictive control, Stability, Mobile robot and tracking trajectory

\section{Introduction}

Model predictive control or a receding horizon control has become an attractive feedback strategy during recent years. Generalized predictive control (GPC) of linear systems has found successful applications, especially in the process industries, due to its robustness to parameter uncertainties (Boucher and Dumar, 1996). However, many systems are, in general, inherently nonlinear. Thus, linear models are often inadequate to describe the process dynamics and nonlinear models have to be used. Much effort has been made to extend GPC to nonlinear systems (Michalska and Mayne, 1993). However, in model predictive control, a nonlinear optimization problem must be solved online with computational complexity (Henson and Seborg, 1997). Therefore, this kind of control law can be applied only for systems with slow dynamics (for example chemical process). To avoid this, several nonlinear predictive laws have been developed (Ping, 1995; Singh, et al. 1995; Souroukh and Kravaris, 1996) where the one-step ahead predictive error is obtained by expanding the output signal and reference signal in a $\mathrm{r}_{i}^{\text {th }}$ order Taylor series, and $\mathrm{r}_{i}^{\text {th }}$ is the relative degree of the $\mathrm{i}^{\text {th }}$ output. Then, the continuous minimization of the predicted

*Corresponding author’s e-mail: hedjar@ccis.ksu.edu.sa tracking errors is used to derive the control law. This paper examines the nonlinear continuous-time generalized predictive control approach based on a finite horizon dynamic minimization of predicted tracking errors with end point constraints to achieve tracking objectives. It is noticed that, the proposed approach increases the tracking performance with regards to Ping's Lu method (Ping, 1995) and Chen's method (Chen, et al. 2003). Indeed, the two performance indexes used by Ping (1995) and Chen, et al. (2003) are combined (Control Integration) to increase the dynamic of the tracking error. Moreover, the recent Chen et al. method given in Chen, et al. ( 2003) assumes that decoupling matrix is not singular, which is not practical. With the proposed combination control, the assumption above will be weakened. It will be shown that some advantages of this control scheme include good tracking performance, clear physical meaning of maximum and minimum control values when saturation occurs with regard to input-output linearization method.

Minimizing a predictive cost function derives the proposed nonlinear predictive controller. A mobile robot is used as an illustrative example to show the tracking performance achieved by this nonlinear predictive controller. Note that Taylor approximation is used to overcome the computation burden.

The rest of the paper is organized as follows: In section 2 , the problem statement is given; a control law is devel- 
oped to minimize the difference between predicted and desired response. The properties of the control law are discussed, including stability. In section 3, the proposed controller is applied to the planning motion problem of the mobile robot. The results are summarized in section 4 where some directions for related research are also provided.

\section{Model Formulation}

\subsection{Optimal Nonlinear Predictive Control}

In the receding horizon control strategy, the following control problem is solved at each $\mathrm{t}>0$ and $\mathrm{x}(\mathrm{t})$ :

$$
\begin{aligned}
& \text { Min } J(x(t), u(t), t)=\underset{u(t)}{\operatorname{Min}} \frac{1}{2} \\
& \int_{t}^{t+h}\left[x(\tau)^{T} Q x(\tau)+u(\tau)^{T} R u(\tau)\right] d \tau
\end{aligned}
$$

subject to the state equation:

$\dot{\mathrm{x}}(\mathrm{t})=\mathrm{f}(\mathrm{x})+\mathrm{g}(\mathrm{x}) \mathrm{u}(\mathrm{t})$

and the terminal end point constraint $\mathrm{x}(\mathrm{t}+\mathrm{h})=0$ for some $\mathrm{h}>0$, where $\mathrm{x}(\mathrm{t}) \subset \mathrm{X} \in \mathfrak{R}^{\mathrm{n}}$ is the state vector, $\mathrm{u}(\mathrm{t})$ $\subset \mathrm{U} \in \mathfrak{R}^{\mathrm{m}}$ represents the control vector. The functions $\mathrm{f}(\mathrm{x}): \mathfrak{R}^{\mathrm{n}} \rightarrow \mathfrak{R}^{\mathrm{n}}$ and $\mathrm{g}(\mathrm{x}): \mathfrak{R}^{\mathrm{n}} \rightarrow \mathfrak{R}^{\mathrm{n} \times \mathrm{m}}$ are sufficiently differentiable, $\mathrm{Q}$ is positive definite matrix and $\mathrm{R}$ positive semi-definite matrix. We denote the optimal control vector to the above problem by: $\mathrm{u}^{*}(\tau), \tau \in[t$, $t+h$ ]. The currently applied control $\mathrm{u}(\mathrm{t})$ is set equal to $\mathrm{u}^{*}(\tau)$. This process is repeated for every next $t$ for stabilization of the system at the origin. However, solving a nonlinear dynamic optimization problem with equality constraints is highly computationally intensive, and in many cases it is impossible to be performed within a reasonable time limit. Furthermore, the global optimization solution cannot be guaranteed in each optimization procedure since, in general, it is a non convex, constrained nonlinear optimization problem (Henson and Seborg, 1997). To overcome these kinds of problems, Ping (1995) and Chen, et al. (2003) proposed to use Taylor approximation.

On one hand, the objective function used by Ping is the one step predicted tracking error given by:

$$
\mathrm{J}_{1}=\frac{1}{2}\|\mathrm{e}(\mathrm{t}+\mathrm{h})\|_{\mathrm{Q}}^{2}+\frac{1}{2}\|\mathrm{u}(\mathrm{t})\|_{\mathrm{R}}^{2}
$$

On the other hand, the cost function used by Chen et al. (2003) is the predicted tracking error over a fixed horizon without control penalty, and is given by:

$\mathrm{J}_{2}=\frac{1}{2} \int_{0}^{\mathrm{h}}\|\mathrm{e}(\mathrm{t}+\tau)\|_{\mathrm{Q}}^{2} \mathrm{~d} \tau$

It is noticed that the derived optimal control of the above cost function increases the dynamics of the tracking error with regard to Ping's method, but the decoupling matrix should be nonsingular.
In this paper, to maximize the tracking performance of previous methods and overcome the assumption of the decoupling matrix, we propose the integration of these two control structures (Control Integration), and the cost function to be minimized is rewritten as:

$\mathrm{J}=\frac{1}{2}\|\mathrm{e}(\mathrm{t}+\mathrm{h})\|_{\mathrm{Q} 1}^{2}+\frac{1}{2} \int_{0}^{\mathrm{h}}\|\mathrm{e}(\mathrm{t}+\tau)\|_{\mathrm{Q} 2}^{2} \mathrm{~d} \tau+\frac{1}{2}\|\mathrm{u}(\mathrm{t})\|_{\mathrm{R}}^{2}$

where $\mathrm{Q}_{1}$ and $\mathrm{Q}_{2}$ are positive definite matrices respectively and $\mathrm{R}$ positive semi-definite matrix.

The desired trajectory is specified by a smooth vector function $\mathrm{x}_{\text {ref }}(\mathrm{t}) \in \mathfrak{R}^{\mathrm{n}}$ for $t \in\left[t_{0}, t_{\mathrm{f}}\right]$. The problem consists in elaborating a control law $\mathrm{u}(\mathrm{x}, t)$ that improves tracking accuracy along the interval [t, $t+h]$, where $h>0$ is a prediction horizon, such that $\mathrm{x}(t+h)$ tracks $\mathrm{x}_{\text {ref }}(t+h)$. That is, the tracking error is defined by:

$e(t+h)=x(t+h)-x_{\text {ref }}(t+h)$

A simple and effective way to predict the influence of $\mathrm{u}(t)$ on $\mathrm{x}(t+h)$ is to use $\mathrm{r}_{i}^{\text {th }}$ order Taylor series expansion, in such a way as to obtain for each component of the vector:

$$
\begin{aligned}
x_{i}(t+h)= & x_{i}(t)+h L_{f}^{0}\left(f_{i}\right)+\frac{h^{2}}{2} L_{f}\left(f_{i}\right)+\cdots \\
& +\frac{h^{r i}}{r_{i} !} L_{f}^{r_{f}^{i}-1}\left(f_{i}\right)+\frac{h^{r^{i}}}{r_{i} !} L_{g} L_{f}^{r_{f}^{i}-2}\left(f_{i}\right) u(t)
\end{aligned}
$$

The predicted state variable in compact form is given by:

$$
\begin{aligned}
& x(t+h)=x(t)+Z(x, h)+\ddot{E}(h) W(x) u(t) \\
& \mathrm{Z}(\mathrm{x}, \mathrm{h})=\left[\begin{array}{llll}
\mathrm{z}_{1}(\mathrm{x}, \mathrm{h}) & \mathrm{z}_{2}(\mathrm{x}, \mathrm{h}) & \cdots & \mathrm{z}_{\mathrm{n}}(\mathrm{x}, \mathrm{h})
\end{array}\right]^{\mathrm{T}} ; \\
& \ddot{E}(h)=\operatorname{diag}\left(\frac{h^{r 1}}{r_{1} !} \cdots \frac{h^{r_{n}}}{r_{n} !}\right) ; \quad W(x)=\left[\begin{array}{llll}
w_{1} & w_{2} & \cdots & w_{n}
\end{array}\right]^{T} \\
& \text { with } z_{i}(x, h)=h f_{i}+\frac{h^{2}}{2} L_{f} f_{i}+\ldots \ldots .+\frac{h^{r i}}{r_{i} !} L_{f}^{r i-1} f_{i} \text { and } \\
& w_{i}=\left[\begin{array}{lll}
L_{g 1} L_{f}^{r i-2} f_{i} & \cdots & L_{g m} L_{f}{ }^{r i-2} f_{i}
\end{array}\right] \text { for } i=1, \ldots, n .
\end{aligned}
$$

where

We also expand each component of $\mathrm{x}_{\mathrm{ref}}(t+h)$ in $\mathrm{r}_{\mathrm{i}}^{\text {th }}$ order Taylor series to have:

$\mathrm{x}_{\text {ref }}(\mathrm{t}+\mathrm{h})=\mathrm{x}_{\text {ref }}(\mathrm{t})+\mathrm{d}(\mathrm{t}, \mathrm{h})$

where $d(t, h)=\left[\begin{array}{llll}d_{1} & d_{2} & \ldots & d_{n}\end{array}\right]^{T}$ with

$\mathrm{d}_{\mathrm{i}}=\mathrm{h} \dot{\mathrm{x}}_{\text {refi }}+\frac{\mathrm{h}^{2}}{2} \ddot{\mathrm{x}}_{\text {ref } \mathrm{i}}+\ldots \ldots \ldots+\frac{\mathrm{h}^{\mathrm{ri}} \quad\left(\mathrm{ri}^{\mathrm{i}}\right)}{\mathrm{r}_{\mathrm{i}} !} \mathrm{x}_{\text {ref } \mathrm{i}}$ 
The tracking error at the next instant $(t+h)$ is then predicted as a function of $\mathrm{u}(t)$ by:

$$
\begin{aligned}
e(t+h) & =x(t+h)-x_{\text {ref }}(t+h)=e(t)+Z(x, h) \\
& -d(t, h)+\ddot{E}(h) W(x) u(t)
\end{aligned}
$$

In order to find the current control $\mathrm{u}(t)$ that improves tracking error along a fixed interval and to avoid the computational burden, the expression of the above predicted tracking error is used in the objective function Eq. (5). Therefore, the unique control signal $u{ }_{\text {op }}$ that minimizes $J$, obtained by setting $\frac{\partial \mathrm{J}}{\partial \mathrm{u}}=0$, is (see appendix):

$$
\begin{aligned}
\mathrm{u}_{\text {op }}= & -\left(\mathrm{W}(\mathrm{x})^{\mathrm{T}} \tilde{\mathrm{A}}(\mathrm{h}) \mathrm{W}(\mathrm{x})+\mathrm{R}\right)^{-1} \\
& \mathrm{~W}(\mathrm{x})^{\mathrm{T}}\left(\mathrm{K}(\mathrm{h}) \mathrm{e}(\mathrm{t})+\mathrm{V}\left(\mathrm{x}, \mathrm{x}_{\text {ref }}, \mathrm{h}\right)\right)
\end{aligned}
$$

where

$$
\begin{aligned}
& \mathrm{e}(\mathrm{t})=\mathrm{x}(\mathrm{t})-\mathrm{x}_{\text {ref }}(\mathrm{t}) ; \mathrm{K}(\mathrm{h})=\ddot{\mathrm{E}}(\mathrm{h})^{\mathrm{T}} \mathrm{Q}_{1}+\int_{0}^{\mathrm{h}} \ddot{\mathrm{E}}(\mathrm{T}) \mathrm{Q}_{2} \mathrm{dT} ; \\
& \tilde{\mathrm{A}}(\mathrm{h})=\ddot{\mathrm{E}}^{\mathrm{T}}(\mathrm{h}) \mathrm{Q}_{1} \ddot{E}^{\mathrm{T}}(\mathrm{h})+\int_{0}^{\mathrm{h}} \ddot{E}^{\mathrm{T}}(\mathrm{T}) \mathrm{Q}_{2} \ddot{\mathrm{E}}(\mathrm{T}) \mathrm{dT} ; \\
& V\left(\mathrm{x}, \mathrm{x}_{\mathrm{ref}}, \mathrm{h}\right)=\ddot{\mathrm{E}}^{\mathrm{T}}(\mathrm{h}) \mathrm{Q}_{1}(\mathrm{Z}(\mathrm{x}, \mathrm{h})-\mathrm{d}(\mathrm{t}, \mathrm{h})) \\
& \quad+\int_{0}^{\mathrm{h}} \ddot{\mathrm{E}}^{\mathrm{T}}(\mathrm{T}) \mathrm{Q}_{2}(\mathrm{Z}(\mathrm{x}, \mathrm{T})-\mathrm{d}(\mathrm{t}, \mathrm{T})) \mathrm{dT}
\end{aligned}
$$

\subsection{Tracking Performances}

We assume that the matrix $\mathrm{W}(x)$ is of full rank. This assumption is needed for the stability analysis, but not necessary for the control law to be applicable, since one can always choose $\mathrm{R}>0$, then the inverse matrix in Eq. (11) will still exist. Let $R=0$, then Eq. (11) becomes:

$$
\mathrm{u}_{\mathrm{op}}=-\mathrm{W}(\mathrm{x})^{-1} \tilde{\mathrm{A}}^{-1}(\mathrm{~h})\left(\mathrm{K}(\mathrm{h}) \mathrm{e}(\mathrm{t})+\mathrm{V}\left(\mathrm{x}, \mathrm{x}_{\mathrm{ref}}, \mathrm{h}\right)\right)
$$

Let $\mathrm{Q}_{1}=\mathrm{q}_{1}$ In and $\mathrm{Q}^{2}=\mathrm{q}_{2} \mathrm{I}_{\mathrm{n}}$, then the dynamic of the closed loop equation of the $\mathrm{i}^{\text {th }}$ component of tracking error vector $\mathrm{e}(\mathrm{t})$ is given in compact form by:

$$
\sum_{j=0}^{r i}\left(q_{1}+\frac{q_{2} h}{j+r_{i}+1}\right) \frac{h^{j}}{j !} e_{i}^{(j)}=0
$$

where

$$
\stackrel{(j)}{e_{i}}=L_{f}{ }^{j-1} f_{i}-\stackrel{(j)}{x} \text { ref i }
$$

for $0<j<r_{i}$ and $\stackrel{(0)}{e} e_{i}=e_{i}=x_{i}-x_{\text {ref } i}$ for $j=0$.

The error dynamics Eq. (13) are linear and time invariant. Thus, the proposed controller that minimizes the predicted tracking error naturally leads to a special case of input/state linearization. The advantage of this controller with regards to the linearization method is a clear physical meaning of maximum and minimum when saturation occurs. Note that, by using Routh Criterion, we can show that the tracking error dynamics Eq. (13) are stable only for systems with $\mathrm{r}_{\mathrm{i}} \leq 4$.
For most mechanical systems with actuator dynamics neglected, the relative degree is $r_{i}=1$ or $r_{i}=2$. In the case where $r_{i}=1$, the eigenvalue of the characteristic equation of the error dynamic is:

口 Ping's method (Ping,1995): $\mathrm{s}_{0}=-\frac{1}{\mathrm{~h}}$.

a Chen et al. (2003): $\mathrm{s}_{0}=-\frac{3}{2 \mathrm{~h}}$

Proposed method: $\mathrm{s}_{0}=-\frac{\left(\mathrm{q}_{1}+\frac{\mathrm{q}_{2} \mathrm{~h}}{2}\right)}{\left(\mathrm{q}_{1}+\frac{\mathrm{q}_{2} \mathrm{~h}}{3}\right) \mathrm{h}}$.

We conclude that the proposed controller achieves faster tracking error dynamic with regards to Ping, (1995) or Chen et al. (2003) method.

\section{Simulation Example}

In this section, the reference trajectory-tracking problem of mobile robot is simulated to show the validity and achieved performance of the proposed method. A kinematics model of a wheeled mobile robot with two degrees of freedom is given by Kim, et al. (2003):

$$
\left\{\begin{array}{l}
\dot{\mathrm{x}}_{\text {ref }}=\mathrm{v}_{\text {ref }} \cos \left(\theta_{\text {ref }}\right) \\
\dot{\mathrm{y}}_{\text {ref }}=\mathrm{v}_{\text {ref }} \sin \left(\theta_{\text {ref }}\right) \\
\dot{\theta}_{\text {ref }}=\omega_{\text {ref }}
\end{array}\right.
$$

where the forward velocity $v$ and the angular velocity $\omega$ are considered as the inputs, $(x, y)$ is the center of the rear axis of vehicle, and $\theta$ is the angle between heading direction and $x$-axis. $d$ is the distance from the origin coordinate of the mobile robot to the axis of the driving wheel.

The nonholonomic constraint is written as the following equation:

$\dot{y} \cos (\theta)-\dot{x} \sin (\theta)=d \theta$

The nonlinear model of the mobile robot can be rewritten as:

$\mathrm{Z}=\mathrm{G}(\theta) \mathrm{U}$

where

$$
\mathrm{Z}=\left[\begin{array}{lll}
\mathrm{x} & \mathrm{y} & \theta
\end{array}\right]^{\mathrm{\Gamma}} ; \mathrm{G}(\theta)=\left|\begin{array}{cc}
\cos (\theta) & -\mathrm{d} \sin (\theta) \\
\sin (\theta) & \mathrm{d} \cos (\theta) \\
0 & 1
\end{array}\right|
$$

and $\mathrm{U}=\left[\begin{array}{ll}\mathrm{v} & \omega\end{array}\right]^{\mathrm{T}}$.

Note that the above model matches the multi-variable affine nonlinear system given by Eq. (2) with $f(x)=0$.

Consider the problem of tracking a reference trajectory given by the equations: 
$\left\{\begin{array}{l}\dot{\mathrm{x}}_{\text {ref }}=\mathrm{v}_{\text {ref }} \cos \left(\theta_{\text {ref }}\right) \\ \dot{y}_{\text {ref }}=v_{\text {ref }} \sin \left(\theta_{\text {ref }}\right) \\ \dot{\theta}_{\text {ref }}=\omega_{\text {ref }}\end{array}\right.$

The optimal control that minimizes the objective function, Eq. (5), subject to constraints, Eq. (1) is:

$$
\begin{aligned}
\mathrm{U}= & -\frac{1}{\mathrm{~h}}\left[\mathrm{G}^{\mathrm{T}}(\theta)\left(\mathrm{Q}_{1}+\frac{\mathrm{h}}{3} \mathrm{Q}_{2}\right) \mathrm{G}(\theta)+\mathrm{h}^{-2} \mathrm{R}\right]^{-1} \\
& \mathrm{G}^{\mathrm{T}}(\theta)\left[\left(\mathrm{Q}_{1}+\frac{\mathrm{h}}{2} \mathrm{Q}_{2}\right) \mathrm{e}(\mathrm{t})-\mathrm{h}\left(\mathrm{Q}_{1}+\frac{\mathrm{h}}{3} \mathrm{Q}_{2}\right) \dot{\mathrm{Z}}_{\text {ref }}(\mathrm{t})\right]
\end{aligned}
$$

In the simulation, the control parameters are chosen as:

$h=0.002, \mathrm{Q}_{1}=\mathrm{Q}_{2}=500 \mathrm{I}_{3}, \quad \mathrm{R}=10^{-3} \mathrm{I}_{2}$.

The reference model and initial conditions are:

$$
\begin{gathered}
\omega_{\mathrm{ref}}=4 \mathrm{rad} / \mathrm{s}, d=1 \mathrm{~m}, v_{\mathrm{ref}}=30 \mathrm{~m} / \mathrm{s}, x(0)=0, \\
y(0)=4 \mathrm{~m} \quad \text { and } \quad \theta(0)=-\frac{\pi}{2} \mathrm{rad} .
\end{gathered}
$$

The limitations on control signals are:

$$
|v| \leq 500 \mathrm{~m} / \mathrm{s} \text { and }|\mathrm{w}| \leq 200 \mathrm{rad} / \mathrm{s}
$$

Figures 1 and 2 show the tracking trajectory in the $x y$ plane. Notice how gracefully the mobile robot tracks the reference trajectory in less than one revolution. Figure 3 depicts the position tracking error given by:

where $\mathrm{e}(\mathrm{t})=\mathrm{Z}(\mathrm{t})-\mathrm{Z}_{\text {ref }}(\mathrm{t})$

We can see that the mobile robot tracks the reference trajectory successfully.

It was stated in the introduction that the advantage of the proposed approach with regard to input-output linearization method is a clear physical meaning of maximum and minimum control values when saturation occurs. Figure 4 depicts the control histories $v(\mathrm{t})$ and $w(\mathrm{t})$ with saturation. Note the initial saturation for both controls due to the large tracking error at the beginning.

The control parameters $Q_{1}, Q_{2}$ and $R$ can be chosen as in classical optimal control theory. The choice of the control parameter $h$, on the other hand, affects the rate of convergence and the amplitude of the control signal. Indeed, to bring the control signals inside the saturation limits, one has to increase the value of $h$ (see Fig. 5) and this will increase time response of the system slightly.

\section{Conclusions}

In this paper, optimal nonlinear predictive controller with end point constraints using Taylor approximation is presented and is applied to multi-variable affine nonlinear system. Minimizing a quadratic cost function of the predicted tracking error and the control input derived control laws. One of the main advantages of this control scheme is that it does not require an online optimization and asymptotic tracking of the smooth reference signal is guaranteed. Moreover, the dynamic of the closed loop system is better than the dynamic obtained by both Ping's and Chen's methods.

The proposed controller is applied to planning motion problem of mobile robot under nonholonomic constraints. Finally, we expect that the results presented here can be explored and extended to discrete implementation of this continuous-time predictive controller either through computers or special purpose chips that can run at higher speeds.

Position of the mobile robot and the reference trajactory for $t=[0.03]$

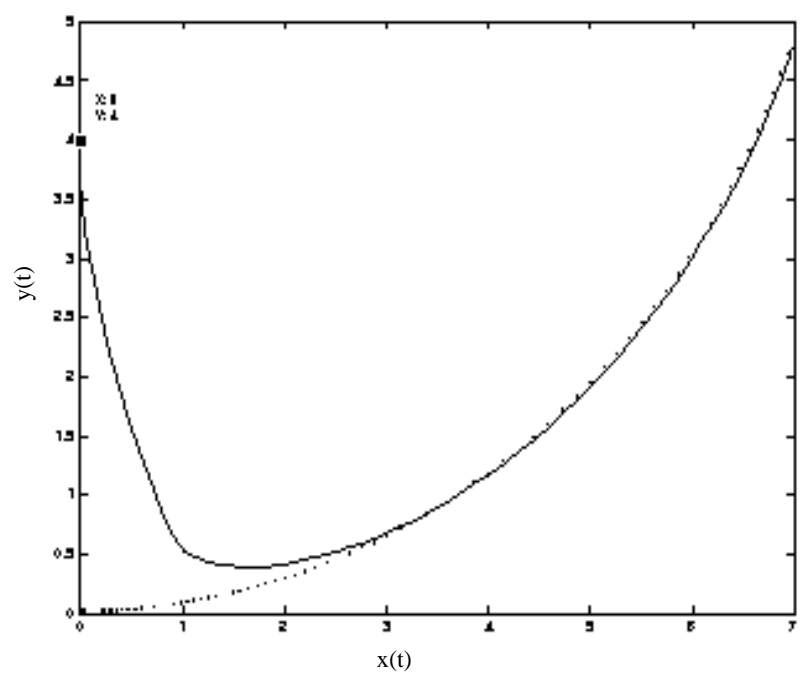

Figure 1. Tracking performance for $t \in[0,0.3 s]$

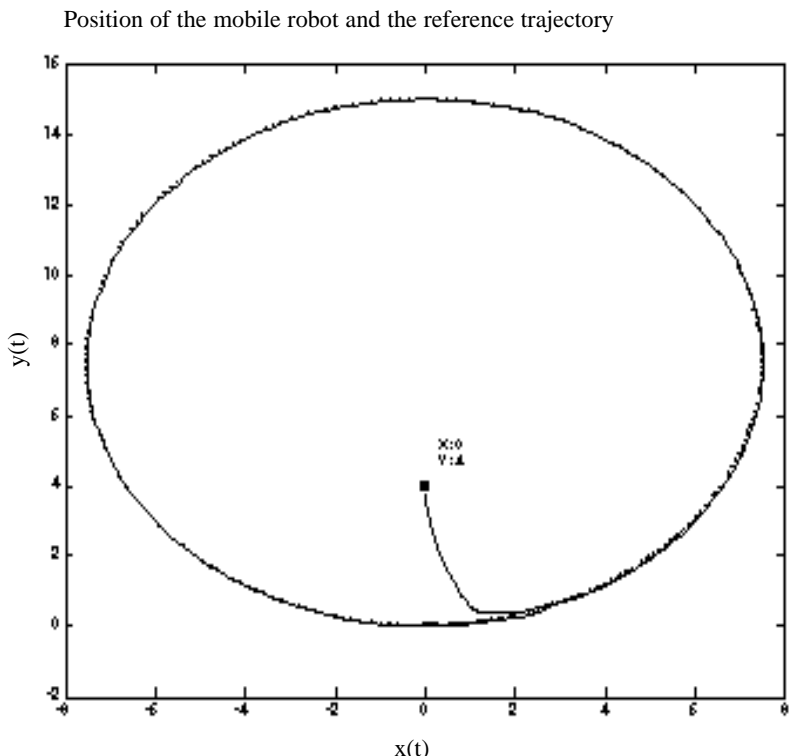

Figure 2. Tracking performance for $t \in[0,2 s]$ 
Tracking Enor:

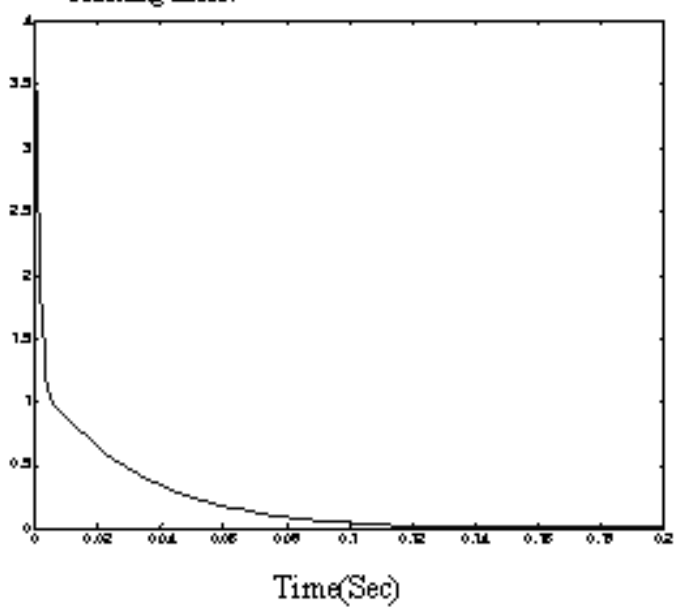

Figure 3. Tracking error
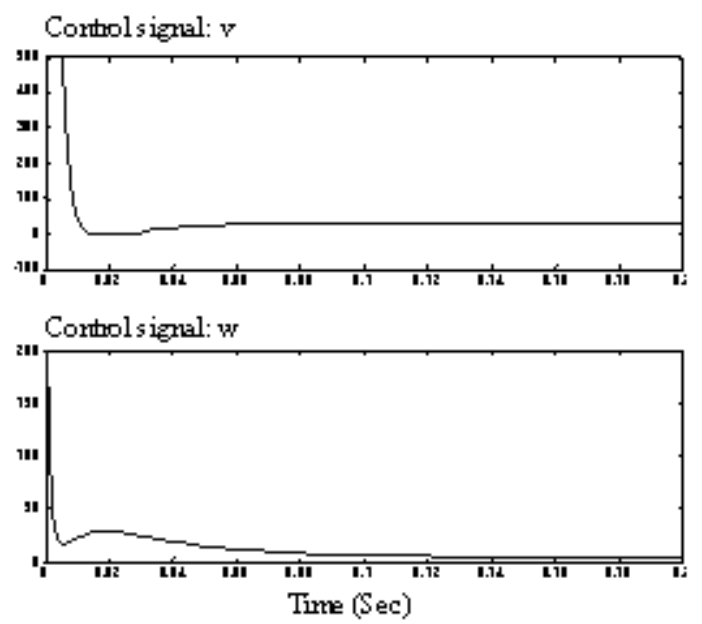

Figure 4. Control signals

Control signal: $\mathrm{v}$
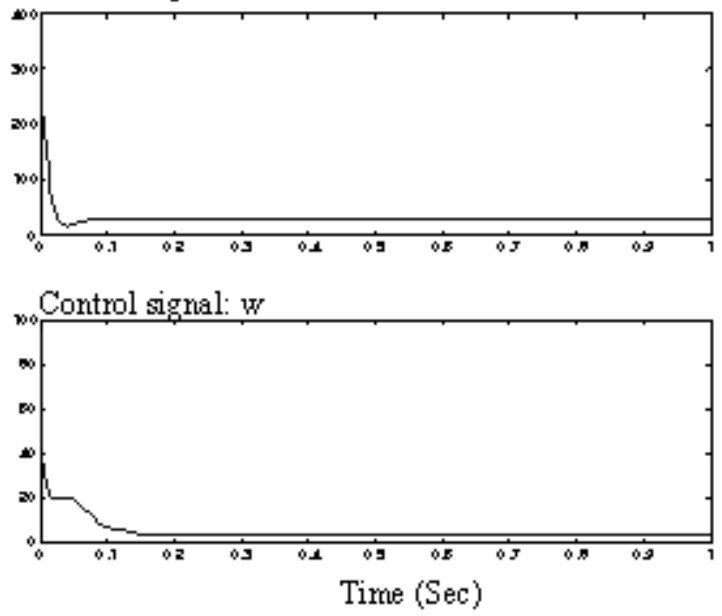

Figure 5. Control signals with $h=0.01$ without saturation

\section{References}

Boucher, P. and Dumur, D., 1966, La commande prédictive, Collection méthode et pratique de l'ingénieur, Edition Technip. Paris.

Chen, W.H., Balance, D.J. and Gawthrop, P.J., 2003, "Optimal Control of Nonlinear Systems: A Predictive Control Approach,” Automatica, Vol. 39, pp. 633-641.

Demircioglu, H. and Gawthrop, P.J., 1991, "Continuous-time Generalized Predictive Control (GPC),” Automatica, Vol. 27(1), pp. 55-74.

Henson, M.A. and Seborg, D.E., 1997, "Nonlinear Process Control," Prentice Hall .

Kim, M.S., Shin, J.H., Hong, S.G. and Lee, J.J., 2003, “Designing a Robust Adaptive Dynamic Controller for Nonholonomic Mobile Robots under Modeling Uncertainty and Disturbances," Mechatronics, Vol. 13, pp. 507-519.

Michalska, H. and Mayne, D.Q., 1993, "Robust Receding Horizon Control of Constrained Nonlinear Systems," IEEE Transactions on Automatic Control, Vol. 38(11), pp. 1623-1633.

Ping, L., 1995, “Optimal Predictive Control of Continuous Nonlinear Systems,” Int. J. of Control, Vol. 62(2), pp. 633-649.

Singh, S.N., Steinberg, M. and DiGirolamo, R.D., 1995, "Nonlinear Predictive Control of Feedback Linearizable Systems and Flight Control System Design,” J. of Guidance, Control and Dynamics , Vol. 18(5), pp. 1023-1028.

Souroukh, M. and Kravaris, C., 1996, “A Continuoustime Formulation of Nonlinear Model Predictive Control," Int. J. of Control, Vol. 63(1), pp. 121146. 
The Journal of Engineering Research Vol. 3, No. 1 (2006) 69-74

\section{Appendix}

$$
\begin{aligned}
J & =\frac{1}{2} e(t+h)^{T} Q_{1} e(t+h)+\frac{1}{2} \int_{0}^{h} e(t+\tau)^{T} Q_{2} e(t+\tau) d \tau \\
& +\frac{1}{2} u^{T}(t) R u(t)
\end{aligned}
$$

By using the predicted tracking error given in Eq. (8), the cost function can be rewritten:

$$
\begin{aligned}
J= & \frac{1}{2}[e+Z(x, h)-d(t, h)+\ddot{E}(h) W(x) u(t)]^{T} \\
& Q_{1}[e+Z(x, h)-d(t, h)+\ddot{E}(h) W(x) u(t)] \\
& +\frac{1}{2} \int_{0}^{h}[e+Z(x, \tau)-d(t, \tau)+\ddot{E}(\tau) W(x) u(t)]^{T} \\
& Q_{2}[e+Z(x, \tau)-d(t, \tau)+\ddot{E}(\tau) W(x) u(t)] d \tau \\
& +\frac{1}{2} u(t)^{T} R u(t) \\
J= & M(e, x, h)+u^{T} W^{T}(x) \\
& {\left[\left(\ddot{E}^{T}(h) Q_{1}+\int_{0}^{h} \ddot{E}{ }^{T}(\tau) Q_{2} d \tau\right) e+\ddot{E}^{T}(h)\right.} \\
& Q_{1}(Z(x, h)-d(t, h)) \\
& \left.+\int_{0}^{h} \ddot{E}^{T}(\tau) Q_{1}(Z(x, \tau)-d(t, \tau)) d \tau\right] \\
& +\frac{1}{2} u^{T}\left[{ }^{T}(x)\left(\ddot{E}^{T}(h) Q_{1} \ddot{E}(h)+\ddot{E}_{0}^{T}(\tau) Q_{2} \ddot{E}(\tau) d \tau\right)\right. \\
& \left.W^{T}(x)+R\right] u . \\
&
\end{aligned}
$$

where $M(e, x, g)$ represents terms that are independent of $u(\mathrm{t})$.

In compact form:

$$
\begin{aligned}
J= & M(e, x, h)+u^{T} W^{T}(x)\left(K(h) e+V\left(x, x_{r e f}, h\right)\right. \\
& +\frac{1}{2} u^{T}\left(W^{T}(x) \tilde{A}(h) W(x)+R\right)
\end{aligned}
$$

where

$$
\begin{aligned}
& e(t)=x(t)-x_{\text {ref }}(t) ; K(h)=\ddot{E}(h)^{T} Q_{1}+\int_{0}^{h} \ddot{E}(T) Q_{2} d T ; \\
& \tilde{A}(h)=\ddot{E}^{T}(h) Q_{1} \ddot{E}(h)+\int_{0}^{h} \ddot{E}^{T}(T) Q_{2} \ddot{E}(T) d T ; \\
& V\left(x, x_{r e f}, h\right)=\ddot{E}^{T}(h) \\
& Q_{1}(Z(x, h)-d(t, h)) \\
& +\int_{0}^{h} \ddot{E}^{T}(T) Q_{2}(Z(x, T)-d(t, T)) d T \\
& u_{o p}=-\left(W(x)^{T} \tilde{A}(h) W(x)+R\right)^{-1} \\
& W(x)^{T}\left(K(h) e(t)+V\left(x, x_{\text {ref }}, h\right)\right)
\end{aligned}
$$

